\title{
Desenvolvimento biológico de Angiostrongylus vasorum (Baillet) Kamensnky (Nematoda, Angiostrongylidae) em Subulina octona Bruguière (Molusca, Subulinidae) em condições de laboratório
}

\author{
Elisabeth Cristina de Almeida Bessa ${ }^{1}$ \\ Walter dos Santos Lima ${ }^{2}$ \\ Erik Daemon ${ }^{3}$ \\ Márcia Cristina Cury ${ }^{2}$ \\ José Luiz de Barros Araújo ${ }^{4}$
}

\begin{abstract}
Biological development of Angiostrongylus vasorum (Baillet) Kamensky (Nematoda, Metastrongylidae) in Subulina octona Bruguière (Mollusca, Subulinidae) in laboratory conditions. With the purpose of investigating the biological aspects of Angiostrongylus vasorum (Baillet, 1866) Kamensky, 1905 in Subulina octona Bruguière, 1789 experimental infections were carried out with the exposure of 90 adult molluses to $155 \mathrm{~L}_{1}$ of the parasite/molluse during 24 hours. The intramolluscan larval development was checked out daily through the chemical digestion of four infected molluscs and one molluscs from the control group during 21 days after infection. Until the $6^{\text {th }}$ day post-infection, only $L_{1}$ of the parasite has been observed. On the $7^{\text {th }}$ day, the presence of $\mathrm{L}_{2}$ was detected and from the $13^{\text {th }}$ day on, $\mathrm{L}_{3}$ of $A$. vasorum also appeared. By means of larval morphometry, it has been observed that, $L_{1}$ had na average size of $335 \times 14,5$ um whereas $L_{2}$ had an average size of $440,7 \times$ 33,9 um on the $7^{\text {th }}$ day ofter infection, reaching a, aximal diameter of 41,8 um on the $12^{\text {th }}$ day after infections. The $\mathrm{L}_{3}$ had an average size of $472,4 \times 38,7 \mathrm{um}$ on the $13^{\text {th }}$ day day and on the $21^{\text {st }}$ day after infection the measure was $551,3 \times 27,6$ um. In order to verify the infectivity of $\mathrm{L}_{3}$, from $S$. octona to the definitive host, infection of a dog was undertaken by means of ingestion of tritured molluses. On the $49^{\text {th }}$ day post-infection, $\mathrm{L}_{1}$ was found in the feaces of the dog and at necropsy on the $84^{\text {th }}$ day post-infection adults worms were found in the lungs.

KEY WORDS. Angiostrongylus vasorum, Subulina octona, intra-mollusc larvae evolution
\end{abstract}

Entre os helmintos que ocorrem no Brasil, observa-se Angiostrongylus vasorum (Baillet, 1866) Kamensky, 1905, (Angiostrongylidae), um nematóide que parasita artérias pulmonares e suas ramificações, ventrículo, átrio e aurícula direita de cães domésticos e carnívoros silvestres.

1) Departamento de Zoologia, Instituto de Ciências Biológicas, Universidade Federal de Juiz de Fora. 36036-330 Juiz de Fora, Minas Gerais, Brasil.

2) Departamento de Parasitologia, Instituto de Ciências Biológicas, Universidade Federal de Minas Gerais. 31270-901 Belo Horizonte, Minas Gerais, Brasil.

3) Departamento de Parasitologia Animal, Instituto de Biologia, Universidade Federal Rural do Rio de Janeiro. 23890-000 Seropédica, Rio de Janeiro, Brasil.

4) Departamento de Parasitologia, Instituto de Patologia Tropical e Saúde Pública, Universidade Federal de Goiás. 74605-050 Goiânia, Gioás, Brasil. 
A principal área endêmica de Angiostrongylus vasorum é o sudoeste da França (DORCHIES 1976), mas relatos de infecções naturais em cães (Canis familiaris Linnaeus, 1758) foram feitos na Austrália (ROBERTS 1940), Espanha (SANCHEZ ACEDO et al. 1979) e Inglaterra (SIMPSON \& NEAL 1982; COBB \& FISCHER $1990 \mathrm{e}$ MARTIN et al. 1993).

No Brasil, casos de cães naturalmente infectados foram descritos por GoNÇALVES (1961) no Rio Grande do Sul, LANGENEGGER et al. (1962) no Rio de Janeiro, GiOVANONNI et al. (1985) no Paraná e LiMA et al. (1985) em Minas Gerais.

A participação de moluscos no ciclo de Angiostrongylus vasorum foi demonstrada pela primeira vez por GuILHON (1960), quando encontrou lesmas do gênero Arion Férrussac, 1819 naturalmente infectadas. Infecções experimentais em moluscos com Angiostrongylus vasorum foram realizadas por GUILHON \& AFGHAHI (1969), GUILHON \& GAALON (1969), ROSEN et al. (1970), ECKERT \& LAMMLER (1972).

No Brasil, não há, até o presente momento, estudos sobre o desenvolvimento intra-molusco de Angiostrongylus vasorum.

Foi verificada, através de infecções experimentais, a evolução larvar de Angiostrongylus vasorum em Subulina octona Bruguère, 1789 (Subulinidae). Esta é uma espécie de molusco terrestre, freqüentemente encontrada, cuja ocorrência foi assinalada em várias partes do mundo, vivendo em jardins próximos aos domicílios humanos, áreas de maior concentração de animais de companhia, entre eles o cão.

Verificou-se ainda, a infectividade de larvas de terceiro estágio de $A$. vasorum provenientes de S. octona, para cães.

\section{MATERIAL E MÉTODOS}

O presente trabalho foi desenvolvido no Laboratório de Malacologia da Universidade Federal Rural do Rio de Janeiro (Seropédica, Rio de Janeiro) e no Laboratório de Helmintologia da Universidade Federal de Minas Gerais, Belo Horizonte, Minas Gerais.

As larvas de primeiro estágio $\left(\mathrm{L}_{1}\right)$ do parasito, utilizadas nas infecções dos moluscos, foram obtidas de cães experimentalmente infectados, pertencentes ao Canil do Instituto de Ciências Biológicas, Universidade Federal de Minas Gerais.

Os exemplares de Subulina octona foram obtidos da criação estoque do Laboratório de Malacologia da Universidade Rural do Rio de Janeiro A manutenção e o manejo dos moluscos foram feitos segundo BESSA \& ARAÚJO (1995a,b). Observações de temperatura (máxima e mínima) e umidade relativa foram feitas diariamente e todas as medições realizadas para os moluscos foram feitas com o auxílio de um paquímetro Kanon (Mardened Stainless $1 / 2$ in 1/20 mm).

Após a coleta, as fezes dos cães experimentalmente infectadas foram colocadas em aparelho de Baermann modificado, composto de um funil de vidro de 13,5 $\mathrm{cm}$ de diâmetro conectada a um tubo de hemólise através de um tubo de borracha contendo água de torneira aquecida a $45^{\circ} \mathrm{C}$. As fezes permaneciam no aparelho por seis horas para recuperação das $\mathrm{L}_{1}$. Após este período, retirava-se o tubo de hemólise, desprezava-se o sobrenadante e, ao sedimento, acrescentava-se água até 
completar três mililitros. Homogeneizava-se o material na tubo de hemólise e desta suspensão retirava-se, com o auxílio de uma pipeta automática (Pipeta 100 microlitos/Pipemat), amostra de 0,1 ml para exame e contagem das larvas. Esta contagem foi feita em uma placa de vidro $(15 \mathrm{~cm}$ de comprimento e $10 \mathrm{~cm}$ de largura) gotejando-se o material sobre a placa, contava-se, com o auxílio de microscópio estereoscópico, todas as larvas presentes no material distribuído sobre a placa. Uma vez verificada a quantidade de $\mathrm{Li}$, presente em $0,1 \mathrm{ml}$, estimava-se a quantidade total de $\mathrm{L}_{1}$, contida nos três mililitros de suspensão, através de regra de três simples. Após este processo, os tubos de hemólise, contendo três $\mathrm{ml}$ de suspensão foram tampados com algodão, etiquetados, embalados em copos plásticos e desta forma transportadas, dentro de uma caixa de polipropileno, para o Laboratório de Malacologia, Universidade Federal Rural do Rio de Janeiro, onde foram feitas as infeccões dos moluscos. O tempo entre a coleta das $\mathrm{L}_{1}$ e as infecções dos moluscos foi de aproximadamente 15 horas.

Para a infecção, os moluscos foram colocados em placas de Petri e sobre eles distribuia-se o material infectante.

Para acompanhar o desenvolvimento larvar intra-molusco realizou-se uma infecção, utilizando-se 90 moluscos adultos, com comprimento de concha medindo entre 9,50 e 13,0 mm. Estes moluscos foram agrupados em duas placas de Petri $(150 \mathrm{X} 20 \mathrm{~mm})$ contendo, cada placa, 45 indivíduos, sobre os quais distribuiu-se a suspensão de larvas. O número de larvas por molusco (por estimativa) foi de 155 e o tempo de exposição foi de 24 horas. O grupo controle continha 30 moluscos, medindo de 9,50 a $12,90 \mathrm{~mm}$, que receberam três $\mathrm{ml}$ de água.

Para a recuperação das larvas $\left(\mathrm{L}_{1}, \mathrm{~L}_{2}, \mathrm{~L}_{3}\right)$ do parasito, foi feita a digestão química dos tecidos dos moluscos segundo WALLACE \& ROSEN (1969). Digeriu-se diariamente, quatro moluscos, infectados e um do grupo controle, durante 21 dias. As digestões foram feitas em temperatura ambiente, durante duas horas, agitando-se o material manualmente. Feito isso, contava-se as larvas, com o auxílio de microscópio estereoscópico.

Para a mensuração do comprimento e largura das larvas de primeiro, segundo e terceiro estágios de Angiostrongylus vasorum, foi utilizado um microscópio óptico com câmara clara embutida, onde a projeção das larvas foi medida. As mensurações foram feitas nos dias em que observou-se crecimento e/ou aparecimento do estágio larvar subseqüente. Quando presentes, foram medidas, além das larvas, as bainhas que as envolviam. Para fixação das larvas, utilizou-se uma gota de lugol.

Para verificar a infectividade de $\mathrm{L}_{3}$ de Angiostrongylus vasorum provenientes de Subulina octona, foi utilizado um cão, SRD, $13 \mathrm{~kg}$, com seis meses e meio de idade, proveniente de Seropédica, Rio de Janeiro, que foi mantido em baia individual, recebendo diariamente ração comercial para cães e água ad libitum. Cerca de 50 dias antes da infecção, o cão foi submetido semanalmente, a exames de fezes, pelo método de Baermann modificado. Após este período, o cão recebeu um lote de 30 moluscos, medindo em média $14,0 \mathrm{~mm}$ de comprimento de concha, previamente infectados ( $248 \mathrm{~L}$ /molusco, com 30 dias de infecção). Os moluscos foram macerados (com concha) e misturados com carne bovina moída, servidos em vasilha plástica, observando-se a completa ingestão da mistura. 
Para se ter uma estimativa do número de larvas utilizadas na inserção do cão, outros cinco moluscos, provenientes da mesmo lote oferecido ao cão, foram digeridas individualmente, $\mathrm{O}$ número de larvas $\left(\mathrm{L}_{3}\right)$ obtido por molusco foi 25,28 , 35,36 , e 46 , perfazendo um total de $170 \mathrm{~L}_{3}$ e uma mèdia de 34 larvas por molusco infectada. Dessa forma, estimou-se que o cão estaria ingerindo entre 750 e $1380 \mathrm{~L}_{3}$ (1020 L 3 em média). Após a infecção, realizou-se, semanalmente, exames de fezes pelo método de Baermann modificado, para pesquisas de larvas de primeiro estágio do parasito.

Para a recuperação dos parasitos adultos, a necrópsia do cão foi feita 84 dias após a infecção. O cão foi sacrificado pela inoculação na veia radial de aproximadamente $10 \mathrm{ml}$ de solução saturada de cloro hidratado.

Para a coleta dos parasitos, os órgãos foram abertos em cubas de vidro (20 x $3 \mathrm{~cm}$ ) contendo solução de $\mathrm{NaCl}$ a $0,85 \%$. Os helmintos encontrados foram recolhidos com auxílio de estiletes e fixados em AFA (álcool-formalina-ácido acético) a $65^{\circ} \mathrm{C}$. Feito isto, os parasitos, machos e fêmeas, foram contados.

Para as fotografias, os helmintos foram montados provisoriamente em lâminas, colocando-se uma gota de ácido acético para auxiliar a melhor visualização das estruturas. Para a realização das fotografias, utilizou-se um microscópio triocular modelo Jenapol com câmara fotográfica modelo mf-AKS $24 \times 36$ Automatic $-2^{3}$.

\section{RESULTADOS E DISCUSSÃO}

Os resultados obtidos após digestão química de exemplares de $S$. octona infectados com A. vasorum constam da tabela I. Demonstra-se ainda na figura 1 a temperatura ambiente observada durante o período de digestão, quando as médias das máximas e mínimas foram, respectivamente 27,09 e $23,29^{\circ} \mathrm{C}$ e a umidade relativa média de $77,9 \%$.

Tabela I. Número total de larvas de Angiostrongylus vasorum e percentual por estágio de desenvolvimento recuperados de quatro exemplares de Subulina octona expostos a 155 $\mathrm{L}_{1} /$ molusco e digeridos diariamente, durante 21 dias após a exposição.

\begin{tabular}{|c|c|c|c|c|}
\hline \multirow{2}{*}{ Dias após infecção } & \multirow{2}{*}{ Número total } & \multicolumn{3}{|c|}{ Larvas recuperadas (\%) } \\
\hline & & $\mathrm{L} 1$ & $\mathrm{~L} 2$ & L3 \\
\hline $1-6$ & 387 & 100,0 & 0,0 & 0,0 \\
\hline 7 & 94 & 30,8 & 69,2 & 0,0 \\
\hline 8 & 197 & 20,8 & 79,2 & 0,0 \\
\hline 9 & 192 & 16,2 & 83,9 & 0,0 \\
\hline 10 & 185 & 10,8 & 89,2 & 0,0 \\
\hline 11 & 194 & 7,7 & 92,3 & 0,0 \\
\hline 12 & 227 & 4,4 & 95,6 & 0,0 \\
\hline 13 & 234 & 4,3 & 89,3 & 6,5 \\
\hline 14 & 184 & 4,9 & 77,7 & 17,4 \\
\hline 15 & 188 & 4,8 & 74,5 & 20,7 \\
\hline 16 & 195 & 3,6 & 48,7 & 47,7 \\
\hline 17 & 216 & 0,9 & 33,8 & 65,3 \\
\hline 18 & 194 & 0,5 & 14,9 & 84,5 \\
\hline 19 & 217 & 0,5 & 8,8 & 90,8 \\
\hline 20 & 216 & 0,0 & 6,9 & 93,1 \\
\hline 21 & 228 & 0,0 & 4,4 & 95,6 \\
\hline
\end{tabular}




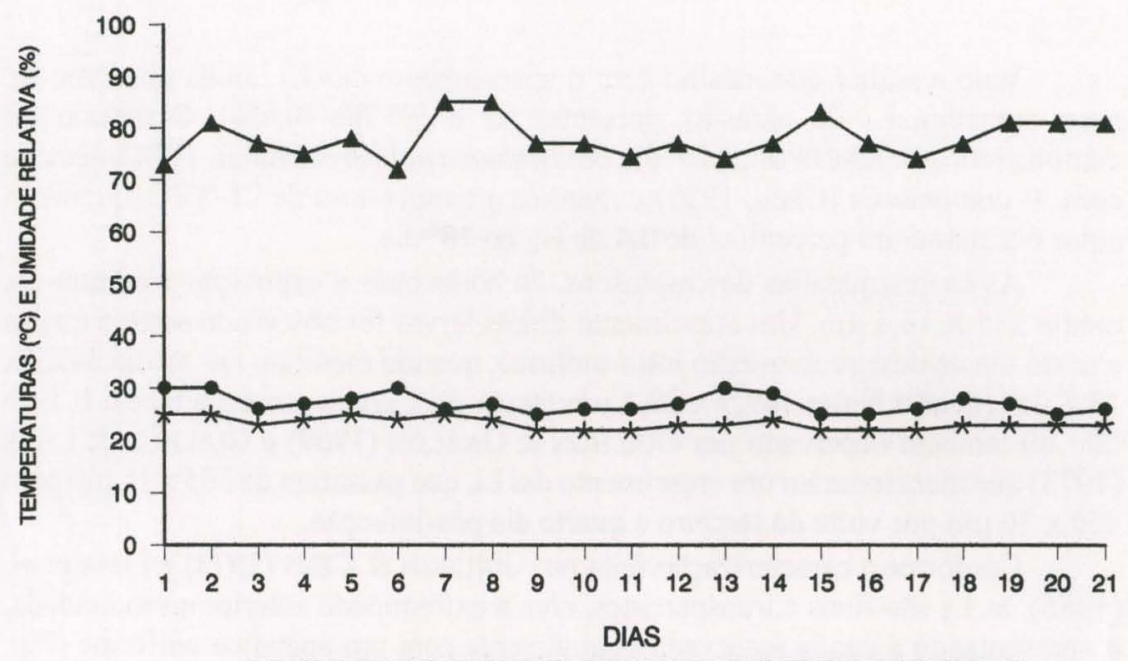

\section{- $\mathrm{T}^{\circ} \mathrm{C}$ máxima * T$^{\circ} \mathrm{C}$ mínima $\star$ Umidade Relativa}

Fig. 1. Temperaturas máxima e mínima e umidade relativa observadas durante 21 dias de digestão de quatro exemplares de Subulina octona expostos a $155 \mathrm{~L}_{1}$ de Angiostrongylus vasorum/molusco.

Observa-se que até o sexto dia após a exposição foram recuperadas somente larvas de primeiro estágio. No sétimo dia de observação houve um decréscimo no número de larvas deste estágio em consequiência do aparecimento de larvas do segundo estágio quando se observou um percentual de 69,2 de L2. O número de $\mathrm{L}_{2}$ cresceu continuamente atingindo o máximo por volta do $12^{\circ}$ dia quando constatouse um percentual de 95,6 . Este percentual começou a decrescer no $13^{\circ}$ dia após a exposição, em conseqüência do aparecimento das larvas de terceiro estágio Neste dia, obteve-se um percentual de $6,5 \mathrm{de} \mathrm{L}_{3}$, no $16^{\circ}$ dia, este valor ultrapassava $47 \%$, estando em patamares superiores a $95 \%$ no $21^{\circ}$ dia. Os resultados deste trabalho são semelhantes aos de GUILHON \& AFGHAHI (1969) que obtiveram, de moluscos infectados experimentalmente e mantidos em temperatura de 18 a $23^{\circ} \mathrm{C}, \mathrm{L} 3$ do mesmo parasito por volta do $16^{\circ}$ e $17^{\circ}$ dia, embora sem fornecer os números e percentuais de larvas recuperadas.

GUILHON \& GAALON (1969) trabalharam com moluscos dulciaquícolas oriundos da França (mantidos a temperatura de 18 a $20^{\circ} \mathrm{C}$ ) e moluscos provenientes de países de clima tropical (mantidos a temperatura de 25 a $27^{\circ} \mathrm{C}$ ) e obtiveram $\mathrm{L}_{2}$ de $A$. vasorum por volta do sétimo e oitavo dia. Nos moluscos provenientes de países tropicais os autores observaram $\mathrm{L}_{3}$ no $13^{\circ}$ dia após a exposição enquanto que nos moluscos oriundos da França, as $\mathrm{L}_{3}$ foram observadas no 16 e $17^{\circ}$ dia após a exposição. Estes autores observaram que os estágios são absolutamente idênticos àqueles observados anteriormente por outros pesquisadores, mas constataram um período menor de evolução (aparecimento das $\mathrm{L}_{3}$ no $13^{\circ}$ dia após a exposição) o que foi interpretado como sendo devido às diferenças de temperatura ambiente ( 25 a $27^{\circ} \mathrm{C}$ e 18 a $\left.23^{\circ} \mathrm{C}\right)$. 
Vale ressaltar que mesmo com o aparecimento das $\mathrm{L}_{3}$, ainda observou-se, neste trabalho, $\mathrm{L}_{1}$ do parasito, presentes até o $19^{\circ}$ dia $(0,5 \%)$. O mesmo foi demonstrado por RACHFORD (1976) com Lymnaea palustris Muller, 1774 infectada com $A$. cantonensis (Chen, 1935) e mantida a temperatura de $21-25^{\circ} \mathrm{C}$, quando o autor constatou um percentual de $0,4 \mathrm{de} \mathrm{L}_{1}$, no $18^{\circ}$ dia.

As $\mathrm{L}_{1}$ recuperadas dos moluscos, 24 horas após a exposição mediram em média 335 X 14,5 $\mu \mathrm{m}$. Um crescimento dessas larvas foi observado entre o quarto e sexto dia de desenvolvimento intra-molusco, quando mediram em média $342,9 \mathrm{x}$ $18,7 \mu \mathrm{m}$ (quarto dia) e 409,5 x 28,1 $\mu \mathrm{m}$ (sexto dia), como mostra a tabela II Este fato foi também observado por GUILHON \& GAALON (1969) e GUILHON \& CENS (1973) que mencionaram um crescimento das $\mathrm{Li}$, que passaram de $365 \times 16 \mu \mathrm{m}$ para $450 \times 30 \mu \mathrm{m}$ por volta do terceiro e quarto dia pós-infecção.

Conforme a caracterização feita por GUILHON \& CENS (1973) e LIMA et al. (1985), as $\mathrm{L}_{1}$ são finas e transparentes, com a extremidade anterior arrendondada, o apresentando a cauda recurvada ventralmente com um apêndice uniforme (Fig. 2).

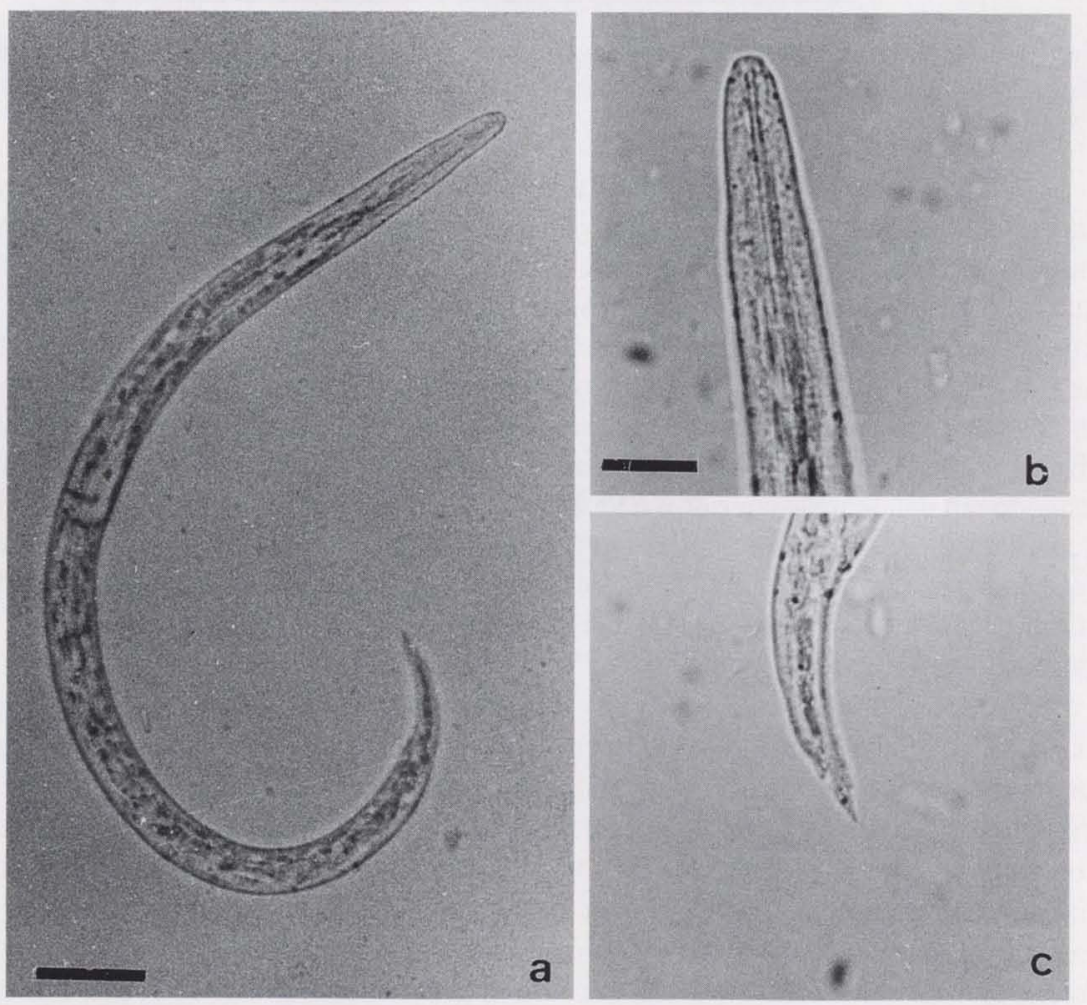

Fig. 2. (a) (Barra $=50 \mu \mathrm{m}$ ) larva de primeiro estágio e Angiostrongylus vasorum (primeiro dia de evolução intra-molusco); (b) (Barra $=30 \mu \mathrm{m}$ ) extremidade anterior; (c) (Barra $=30 \mu \mathrm{m}$ ) detalhe da cauda. 
Tabela II. Comprimento e largura $(\mu \mathrm{m})$ de $L_{1}$ de Angiostrongylus vasorum, com valores máximo, mínimo e médio, desvio padrão (S) e coeficiente de variação (CV) em diferentes dias pós-infecção.

\begin{tabular}{|c|c|c|c|c|c|c|}
\hline \multirow{2}{*}{ Valores } & \multicolumn{3}{|c|}{ Comprimento $(\mu \mathrm{m})$ nos dias pós-infecçăo } & \multicolumn{3}{|c|}{ Largura $(\mu \mathrm{m})$ nos dias pós-infecçăo } \\
\hline & 1 & 4 & 6 & 1 & 4 & 6 \\
\hline Maior & 363,600 & 418,200 & 454,500 & 15,500 & 21,800 & 30,900 \\
\hline Menor & 272,700 & 290,900 & 309,100 & 13,600 & 13,600 & 25,500 \\
\hline Média & 335,000 & 342,900 & 409,500 & 14,500 & 18,700 & 28,100 \\
\hline Desvio padrăo & 23,219 & 37,393 & 41,646 & 0,705 & 3,047 & 2,035 \\
\hline Coeficiente de variação (\%) & 6,931 & 10,905 & 10,170 & 4,861 & 6,295 & 7,243 \\
\hline
\end{tabular}

No sétimo dia, as L2 são pouco móveis e dispostas em arco, dentro do tecido do hospedeiro. A presença de grânulos dentro das células intestinais lhes conferem uma cor marrom. É possível distinguir, em algumas larvas, a primeira bainha que as envolvem. Neste dia as larvas ocupam todo o espaço dentro da bainha. Assim as medidas das larvas coincidem com as medidas das bainhas. As larvas mediram, conforme a tabela III, 418,2 a 454,5 $\mu \mathrm{m} \times 30,0$ a $38,2 \mu \mathrm{m}(\mathrm{x}=440,7 \times 33,9 \mu \mathrm{m})$, valores semelhantes aqueles encontrados por GUILHON \& CENS (1973) que foram de $420-450 \mu \mathrm{m} \times 42 \mu \mathrm{m}$. Por volta do nono dia as larvas começam a se agitar dentro da bainha (Fig. 3) e no $12^{\circ}$ dia elas atingem o diâmetro máxima de $41,8 \mu \mathrm{m}(\mathrm{x}=$ $39,2 \mu \mathrm{m})$ (Tab. III).
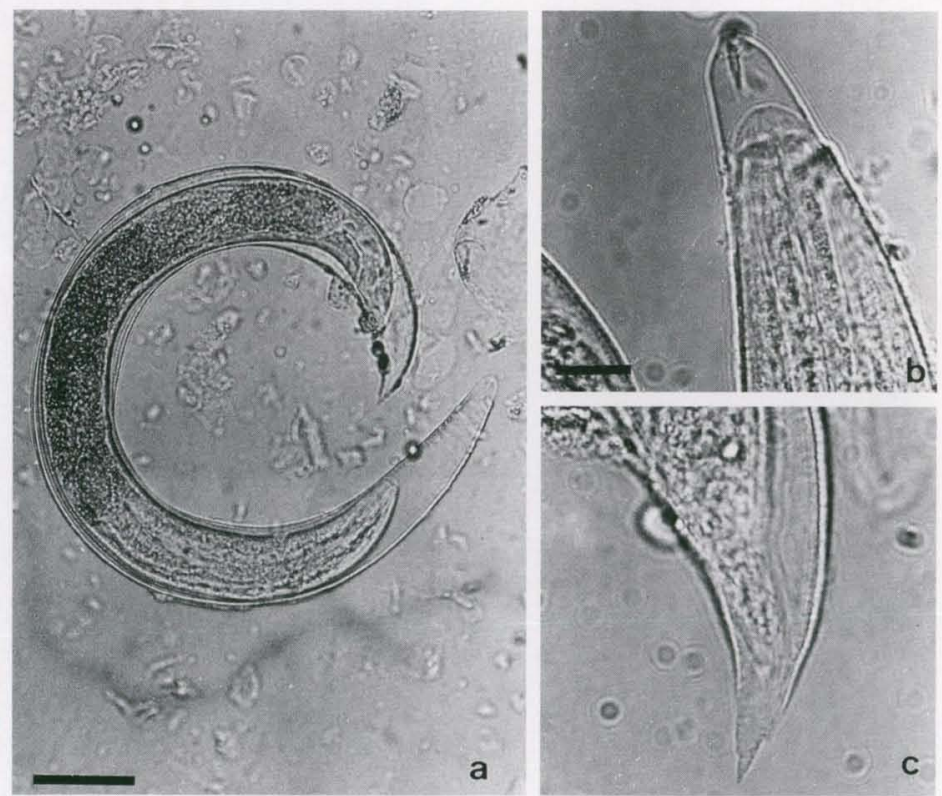

Fig. 3. (a) (Barra $=50 \mu \mathrm{m}$ ) larva de segundo estágio de Angiostrongylus vasorum (nono dia de evolução intra-molusco); (b) (Barra $=10 \mu \mathrm{m}$ ) extremidade anterior; (c) (Barra $=10 \mu \mathrm{m}$ ) extremidade posterior, destacando a bainha da larva. 
Tabela III. Comprimento e largura $(\mu \mathrm{m})$ de L2 de Angiostrongylus vasorum, com valores máximo, mínimo e médio, desvio padrão (S) e coeficiente de variação (CV), para larvas e bainhas, em diferentes dias pós-infecção.

\begin{tabular}{|c|c|c|c|c|c|c|c|c|}
\hline \multirow{3}{*}{ Valores } & \multicolumn{4}{|c|}{ Comprimento $(\mu \mathrm{m})$ nos dias pós-infecçăo } & \multicolumn{4}{|c|}{ Largura $(\mu \mathrm{m})$ nos dias pós-infecçăo } \\
\hline & \multicolumn{2}{|c|}{7} & \multicolumn{2}{|c|}{12} & \multicolumn{2}{|c|}{7} & \multicolumn{2}{|c|}{12} \\
\hline & Larva & Bainha & Larva & Bainha & Larva & Bainha & Larva & Bainha \\
\hline Maior & 454,500 & - & 454,500 & 500,000 & 38,200 & - & 41,800 & 41,800 \\
\hline Menor & 418,200 & - & 418,200 & 436,400 & 30,000 & - & 36,400 & 36,400 \\
\hline Média & 440,700 & - & 451,100 & 486,900 & 33,900 & - & 39,200 & 40,600 \\
\hline Desvio padrăo & 13,367 & - & 17,442 & 17,649 & 2,834 & - & 1,977 & 3,941 \\
\hline Coeficiente de variaçăo (\%) & 3,033 & - & 3,954 & 3,625 & 8,360 & - & 5,044 & 9,707 \\
\hline
\end{tabular}

No $13^{\circ}$ dia de desenvolvimento começam a aparecer as $\mathrm{L}_{3}$ que são móveis no interior de duas bainhas, bem visíveis nas extremidades das larvas (Fig. 4). Por volta do $15^{\circ}$ e $16^{\circ}$ dias, as $\mathrm{L}_{3}$ tornaram-se mais claras e transparentes pois o material de reserva começou a desaparecer das células intestinais. Tornaram-se mais nítidos dois bastões quitinosos, dispostos longitudinalmente, na extremidade anterior das L3.
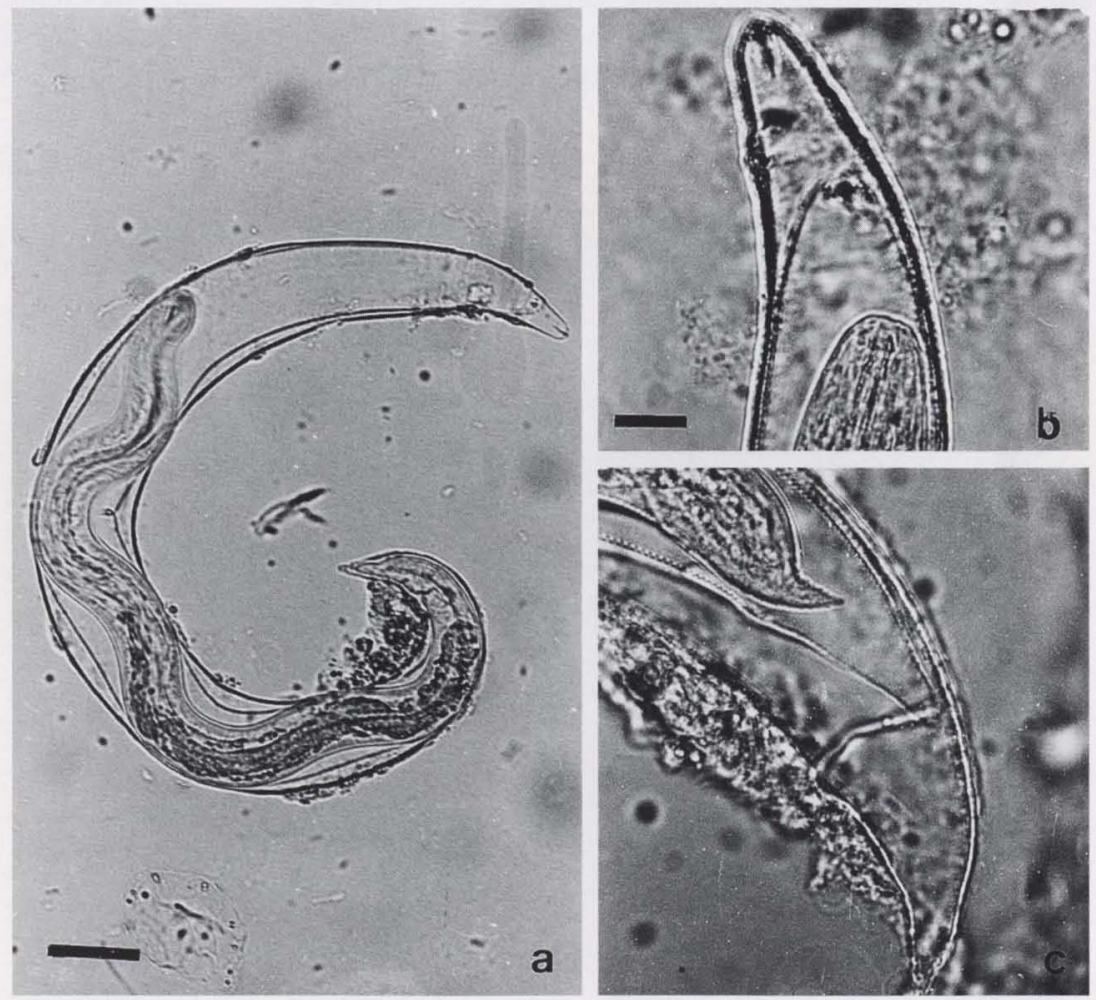

Fig. 4. (a) (Barra $=50 \mu \mathrm{m})$ larva de terceiro estágio de Angiostrongylus vasorum $\left(13^{\circ}\right.$ dia de evolução intra-molusco); (b) (Barra $=10 \mu \mathrm{m}$ ) extremidade anterior; (c) (Barra $=10 \mu \mathrm{m}$ ) extremidade posterior. Destaque para as duas bainhas. 
No $21^{\circ}$ dia quase todas as larvas apareceram livres das bainhas, os bastões quitinosos ficaram evidentes, assim como a cauda da larva, que sempre termina com um apêndice digitiforme (Fig. 5). Neste dia as larvas mediram em média 551,3 x $27,6 \mu \mathrm{m}$ (Tab. IV), Estes valores são semelhantes aqueles encontrados par GUILHON \& GAALON (1969) e GUILHON \& CENS (1973) que foram, respectivamente, $550 \mathrm{x}$ $26 \mu \mathrm{m}$ e $528-590 \times 26-33 \mu \mathrm{m}(\mathrm{x}=555 \times 30 \mu \mathrm{m})$.

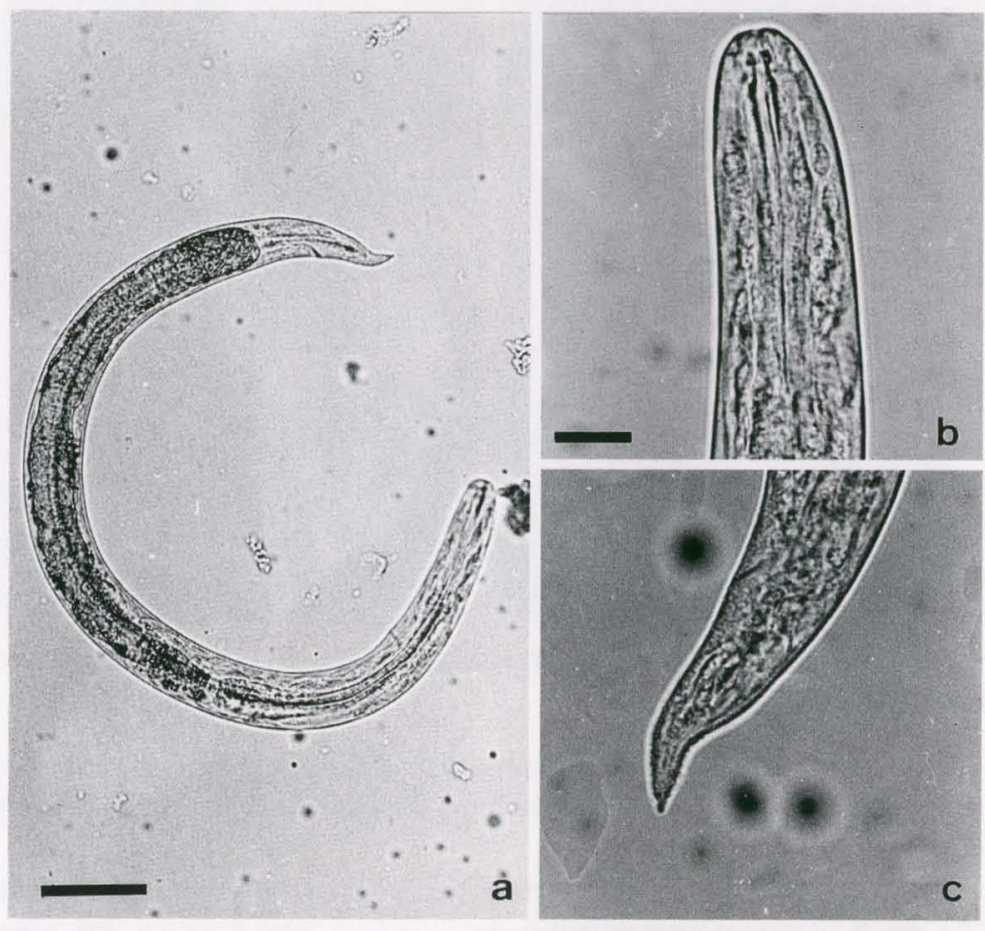

Fig. 5. (a) (Barra $=50 \mu \mathrm{m})$ larva de terceiro estágio de Angiostrongylus vasorum $\left(21^{\circ}\right.$ dia de evolução intra-molusco); (b) (Barra $=10 \mu \mathrm{m}$ ) extremidade anterior, mostrando um par de bastões quitinosos; (c) (Barra $=10 \mu \mathrm{m}$ ) detalhe da cauda.

Tabela IV. Comprimento e largura $(\mu \mathrm{m})$ de $\mathrm{L}_{3}$ de Angiostrongylus vasorum, com valores máximo, mínimo e médio, desvio padrão $(\mathrm{S})$ e coeficiente de variação $(\mathrm{CV})$, para larvas e bainhas, em diferentes dias pós-infecção.

\begin{tabular}{|c|c|c|c|c|c|c|c|c|}
\hline \multirow{3}{*}{ Valores } & \multicolumn{4}{|c|}{ Comprimento $(\mu \mathrm{m})$ nos dias pós-infecção } & \multicolumn{4}{|c|}{ Largura $(\mu \mathrm{m})$ nos dias pós-infecção } \\
\hline & \multicolumn{2}{|c|}{13} & \multicolumn{2}{|c|}{21} & \multicolumn{2}{|c|}{13} & \multicolumn{2}{|c|}{21} \\
\hline & Larva & Bainha & Larva & Bainha & Larva & Bainha & Larva & Bainha \\
\hline Maior & 527,300 & 590,900 & 590,900 & - & 41,800 & 41,800 & 32,700 & - \\
\hline Menor & 418,200 & 527,300 & 509,100 & - & 32,700 & 36,400 & 25,500 & - \\
\hline Média & 472,400 & 554,600 & 551,300 & - & 38,700 & 40,200 & 27,600 & - \\
\hline Desvio padrăo & 32,577 & 26,826 & 26,317 & - & 2,590 & 1,554 & 1,883 & - \\
\hline Coeficiente de variaçăo (\%) & 6,896 & 4,837 & 4,774 & - & 6,694 & 3,866 & 6,823 & - \\
\hline
\end{tabular}



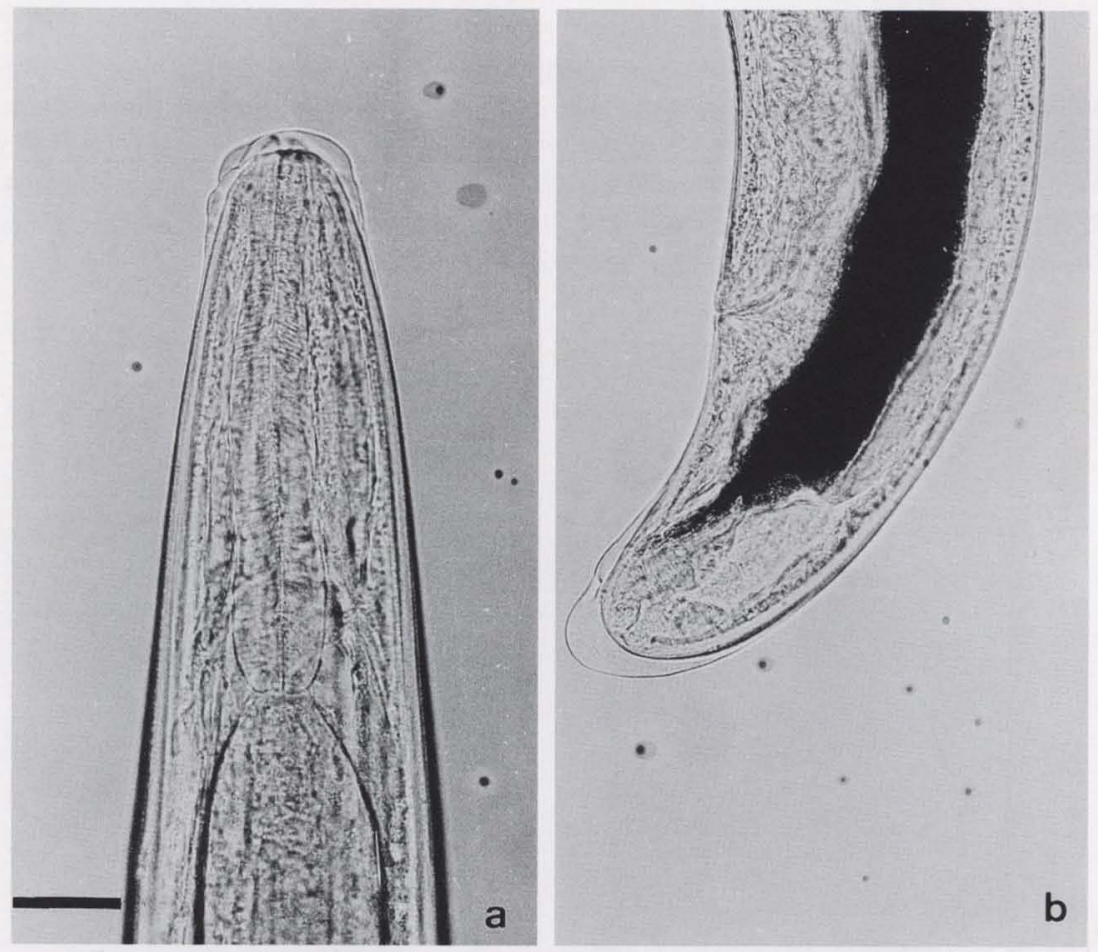

Fig. 6. Angiostrongylus vasorum. (a) Extremidade anterior; (b) extremidade postorerior da fêmea (Barra $=50 \mu \mathrm{m})$.

Vale destacar que a espécie de molusco utilizada, como hospedeiro intermediário, no presente estudo não influenciou a duração do ciclo nem o tamanho das larvas.

Através dos exames semanais das fezes do cão, experimentalmente infectado com $\mathrm{L}_{1}$ de $A$. vasorum provenientes de $S$. octona, constatou-se que o período pré-patente foi de 49 dias. O presente resultado é semelhante àquele encontrado por GUILHON (1960), que observou um período pré-patente de 44-49 dias em cães experimentalmente infectados com $\mathrm{L}_{3}$ provenientes de lesmas de gênero Arion. Utilizando $\mathrm{L}_{3}$ do mesmo parasito, sendo estas larvas provenientes de Helix aspersa Muller, 1744, H. pomatia Linnaeus, 1758 e Cepaeca memoralis Linnaeus, 1758, todos moluscos terrestres, GUILHON \& AFGHAHI (1969) infectaram cães e confirmaram a presença de $\mathrm{L}_{1}$ do parasito nas fezes dos mesmos, 46 a 56 dias após a infecção. ROSEN et al. (1970) observam $\mathrm{L}_{1}$ nas fezes de cães infectados com $\mathrm{L}_{3}$ de A. vasorum, 49 a 60 dias após a infecção e GUILHON \& CENS (1973) observaram um periodo pré-patente de 38 a 50 dias para cães experimentalmente infectados com A. vasorum.

O número estimado de larvas $\left(\mathrm{L}_{1}\right)$ observado nas fezes do cão 49 dias após a infecção foi de 750 . Outros três exames foram feitos antes da necropsia do animal, que ocorreu no $84^{\circ}$ dia pós-infecção no $56^{\circ}$ dia, quando se contou, por estimativa, 
$600 \mathrm{~L}_{1}$; no $63^{\circ}$ dia, $11.250 \mathrm{~L}_{1}$ e no $70^{\circ}$ dia pós-infecção contou-se $15.610 \mathrm{~L}_{1}$. Estes resultados concordam com aqueles obtidos por RoSEN et al. (1970) que, apesar de não terem mencionado o número de $\mathrm{L}_{1}$ observado nem o intervalo entre os exames feitos, verificaram um pequeno número de $\mathrm{L}_{1}$ de $A$. vasorum durante os primeiros dias de patência e um aumento no decorrer dos exames.

O número total de parasitos recuperados durante a necrópsia foi de 362 , dos quais 133 eram machos $(36,7 \%)$ e 229 fêmeas $(63,3 \%)$. Estes resultados são semelhantes aos obtidos por GUILHON (1960) que recuperou, em dois cães experimentalmente infectados com um número de L 3 não mencionada, 1821 espécimes de $A$. vasorum, sendo 615 machos (34\%), 1206 fêmeas (66\%) e 299 parasitos, destes 93 machos $(31,1 \%)$ e 206 fêmeas $(68,9 \%)$, respectivamente.

No que se refere à morfologia dos adultos, constatou-se que, como o observado por LimA et al. (1985), A. vasorum é um nematóide com o corpo delgado e alongado, ligeiramente atenuado nas extremidades. A cutícula é fina e transparente e na extremidade cefálica é, às vezes, ligeiramente dilatada. A abertura bucal é pequena, arrendondada e tem comunicação direta com o esôfago que é clariforme, ligeiramente mais alargado na porção final (Fig. 6a).

A fêmea é maior e mais robusta que o macho. Os ovários são longos. A vulva situa-se anteriormente ao ânus, A cauda é curta e a extremidade posterior é arredondada, apresentando uma expansão cuticular em forma de bainha que se projeta além da cauda (Fig. 6b).

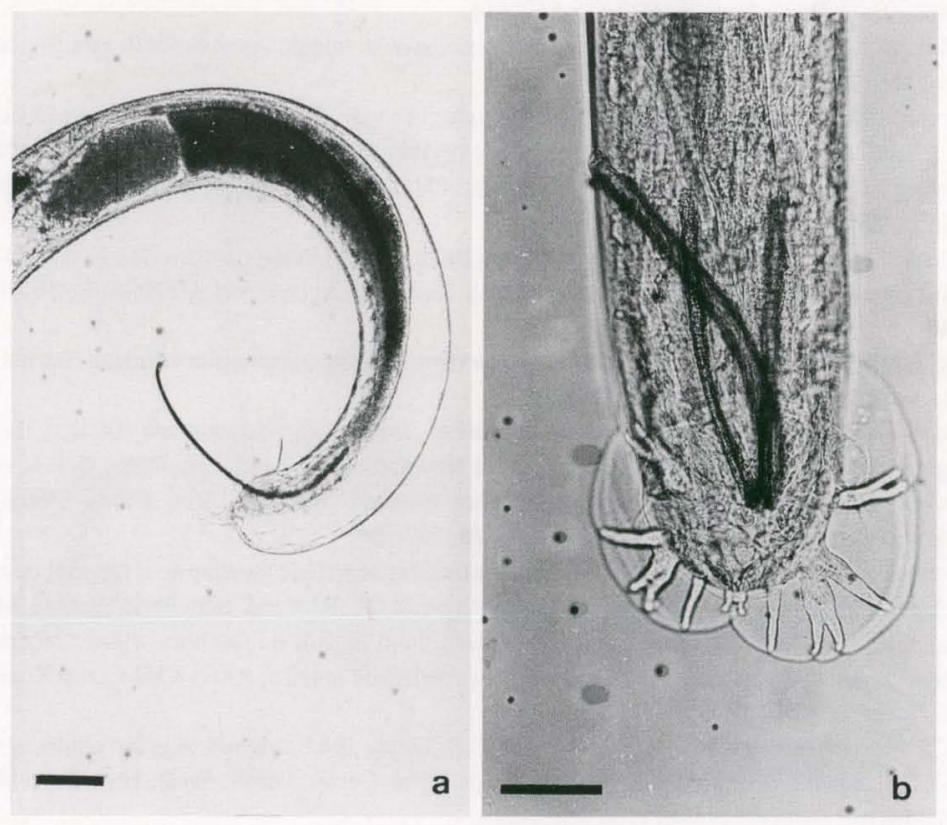

Fig. 7. Angiostrongylus vasorum, extremidade posterior do macho. (a) $($ Barra $=100 \mu \mathrm{m})$ vista lateral mostrando os espículos; (b) (Barra $=50 \mu \mathrm{m}$ ) vista ventral, mostrando a bolsa copuladora. 
Os machos apresentam bolsa copuladora é pequena, porém distinta e característica, desprovida de lobo mediano, com dois lobos laterais idênticos e simétricos. Raios ventrais (ventro-ventral e ventro-lateral) originam-se de um tronco comum, separando-se na extremidade e estendendo-se até quase a borda do lobo. Os raios laterais também originam-se de um tronco comum, mas o lateral anterior torna-se divergente a partir da base, enquanto os raios lateral médio e lateral posterior continuam unidos, separando-se próximo ou no terço final. Os raios dorsais laterais originam-se separadamente do dorsal médio; este é mais curto e grosso e com dois dígitos bem distintos (Fig. 7b). Os espículos são longos, fortes e sub-iguais, com estriações transversais (Fig. 7a,b).

As presentes observações confirmam as descrições feitas por GoNÇALVES (1961), GuILHON \& CENS (1973), GIOVANNONI et al. (1985) e LiMA et al. (1985) para A. vasorum. Em exemplares recém-montados, pode-se observar a presença de gubernáculo; este é navicular e fracamente quitinizado, concordando com as descrições feitas por GUILHON \& CENS (1973) e UBELAKER (1986) para esta estrutura.

\section{REFERÊNCIAS BIBLIOGRÁFICAS}

Bessa, E.C. DE A. \& J.L. DE B. ARAÚJo. 1995a. Ocorrência de autofecundação em Subulina octona (Burguière) (Pulmanata, Subulinidae) sob condições de laboratório. Revta bras. Zool. 2 (3): 719-723.

- 1995b. Oviposição, tamanho de ovos e medida do comprimento da concha em diferentes fases da desenvolvimento de Subulina octona (Burguière) (Pulmonata, Subulinidae) em condições de labaratório Revta bras. zool. 2 (3): 647-654.

COBB, M.A. \& M.A. FISHER. 1990. Angiostrongylus vasorum: transmission in south east England Vet.

Rec. 126: 529.

Dorchies, P. 1976. Etude zoolique, d' Angiostrongylus vasorum. L'Animal de Compagnie 11: 45-45. ECKerT, J. \& G. LAMmLer. 1972. Angiostrongylose bei mensch und tier. Z. Parasitenkd. 39: 303-322. Giovannoni, M.; B.F. Fernandez \& L.C. Kavinski. 1985. Angiostrangilose do cão. Arq. Biol. Tecn., Curitiba, 28: 601-604.

GoNÇALVES, P.C. 1961. Angiostrongylus vasorum (Baillet, 1866), novo parasito do cão no Rio Grande da Sul (Brasil) (Nematoda: Metastrongyloidea). Rev. Fac. Agron. Vet. UFRGS, Porto Alegre, 4: 35-40.

Guilhon, J. 1960. Rôle des limacidés dans le cyde evólutif d' Angiostrongylus vasorum (Baillet, 1866). C.R. Acad. Sc., Paris, 251: 2252-2253.

Guilhon, J. \& A. AfGHAHI. 1969. Évolution larvaire d'Angiostrongylus vasorum (Baillet, 1866) dans l'organisme de diverses espéces de mollusques terrestres. C.R. Acad. Sci., Paris, 268: 434-436.

Guilhon, J. \& B. Cens. 1973. Angiostrongylus vasorum (Baillet, 1866) Etude biologique et morphologique. Ann. Parasit. Hum. Comp. 48: 567-596.

GUILHON, J. \& A. DE GAALON. 1969. Évolution larvaire d'un nématode parasite de l'appariel circulatoire du chien dans l'organisme de mollusques dulçaquicoles. C.R. Acad. Sci., Paris, 268: 612-615.

Langenegger, J.; A.M. Langenegger; P. Dacorso Filho \& G.L.A. Gouvea. 1962. Ocorrência da infestação por Angiostrongylus vasorum em cães do Rio de Janeiro. Anais VIII Cong. Brasil. Vet., Belo Horizonte: 246-247.

Lima, W.S.; H.M.A. CostA; M.P. GuimarÃes \& C.R. LeITE. 1985. Angiostrongylus vasorum (Baillet, 1866) (Nematoda Protostrongylidae) em cães de Minas Gerais, Brasil. Mem. Inst. Oswaldo Cruz 80: 233-235.

Martin, M.W.S.; G. Ashton; V.R. SimpSon \& C. NeAl. 1993. Angiostrongylosis in Corhwall: Clinical presentation of eight cases. Jour. Small Anim. Practice 34: 20-25.

RACHFORD, F.W. 1976. Host-parasite relationship of Angiostrongylus cantonensis in Lymnaea palustris 
I. Intramolluscan Larval Grawth and Development. Exp. Parasit. 39: 377-381.

ROBERTS, F.H.S. 1940, Notes an some helminths infesting domestic animals in Queensland. Austral. Vet. Jour. 16: 30-33.

Rosen, L.; L.A. AsH \& G.D. WaLlace. 1970. Life history of the canine lungworn Angiostrongylus vasorum (Baillet). Amer. Jour. Vet. Res. 31: 131-143.

Sanchez, A.C.; J.B. Diez; J.G. Morales; L.C. Menendes; J.A.C. Hernandes \& J.A.G. Jalon. 1979. Angiostrongylosis canina. Ver. Iber. Parasit. L. 39: 135-142.

SiMPSON, V.R. \& C. NEAL. 1982. Angiostrongylus vasorum infection in dogs and slugs. Vet. Rec. 111: 303-304.

UBELAKER, L.E. 1986. Systematics of especies refered to the genus Angiostrongylus. Jour. Parasit. 72: 237-244.

Wallace, G.D. \& L. Rosen. 1969. Techniques for recovering and identitying larve Angiostrongylus cantonensis from molluscs. Malacologia 7: 427-438.

Recebido em 07.V.1998; aceito em 01.II.2000. 